\title{
Halodehydroxylation of alcohols to yield benzylic and alkyl halides in ionic liquids
}

\author{
Chad F. Petten, Hassan A. Kalviri and Francesca M. Kerton*
}

\begin{abstract}
Background: Alcohols are widely used, and sometimes renewable, reagents but the hydroxyl moiety is a relatively poor leaving group under mild conditions. Direct nucleophilic substitution of alcohols is a desirable reaction for synthetic and process chemists.

Results: Synthesis of twelve alkyl and benzyl halides was achieved in [Bmim]PF ${ }_{6}(\mathrm{Bmim}=1$-butyl-3-methylimidazolium) from their parent alcohols using ammonium halides as the halogenating agents. Trends in reactivity based on the alcohol and halide were discovered. Mechanistic evidence suggests that the reaction proceeds via $\mathrm{S}_{N} 2$ substitution of the hydroxyl group, which is activated via hydrogen-bonding with the acidic proton of the imidazolium cation. Also, for benzyl substrates, equilibria involving formation of dibenzyl ether complicate the reactions and reduce optimum yields.

Conclusions: Ammonium halides are useful, solid and relatively safe reagents for the conversion of some primary alcohols to organohalides in ionic liquids (yields up to $81 \%$ ). Indanol under the same conditions yields biindenylidene (GC yield $63 \%$ ).
\end{abstract}

Keywords: Halogenation, Ionic liquid, Catalysis, Substitution, Solid acid, Microwave, Dehydration

\section{Background}

Alcohols are widely encountered chemicals and are useful intermediates in modern organic synthesis due to their ability to be transformed into a wide range of products. Furthermore, many renewable feedstocks contain hydroxyl moieties. Unfortunately, hydroxyl groups are not favourable leaving groups under mild conditions. This usually means, for nucleophilic substitution reactions, that the alcohol must be activated and subsequently displaced in order to produce the desired product. This sort of procedure violates the principles of green chemistry as the activation step can be considered unnecessary derivatization. Therefore, direct nucleophilic substitution of alcohols is desired and is one of the key green chemistry research areas previously identified by pharmaceutical manufacturers [1]. Catalytic methods for these reactions remain relatively underexplored and were the focus of a recent review [2].

*Correspondence: fkerton@mun.ca

Department of Chemistry, Memorial University of Newfoundland, St. John's, NL A1B 3X7, Canada
In particular, halogenation of alcohols is a useful reaction as alcohol-starting materials are cheap and large varieties are commercially available. Many procedures are known that convert alcohols to halides however, they can involve harsh conditions and hazardous chemicals. For example, the chlorination of alcohols is traditionally performed using $\mathrm{HCl}$ gas or thionyl chloride, both of which are hazardous to human health and often produce many side reactions. Although improvements have been made upon these halogenation reactions, further green methodology is desired. On a laboratory scale, ammonium halide salts $\left(\mathrm{NH}_{4} \mathrm{X}\right)$ are solid reagents, which means they are easy to handle and measure.

Discovering a mild, neutral, highly selective and environmentally friendly system for catalytic dehydroxyhalogenation is desirable but it is not a trivial task. Compromises in the journey towards an ideal sustainable process have to be made. This is exhibited by some of the trends found in the literature regarding chlorination of alcohols. Some reports involve the use of triphenylphosphine $\left(\mathrm{PPh}_{3}\right)$ as a superstoichiometric additive in dehydroxychlorination reactions [3, 4]. Although these

\section{Chemistry Central}

(C) 2015 Petten et al. This article is distributed under the terms of the Creative Commons Attribution 4.0 International License (http://creativecommons.org/licenses/by/4.0/), which permits unrestricted use, distribution, and reproduction in any medium, provided you give appropriate credit to the original author(s) and the source, provide a link to the Creative Commons license, and indicate if changes were made. The Creative Commons Public Domain Dedication waiver (http://creativecommons.org/ publicdomain/zero/1.0/) applies to the data made available in this article, unless otherwise stated. 
systems use mild conditions (often performed at room temperature with neutral chlorinating agents), exhibit high selectivity, and possess a large and diverse substrate scope, a large amount of $\mathrm{PPh}_{3}$ oxide is formed in the reaction, which causes purification to be troublesome. An interesting reagent that has been researched in dehydroxychlorination reactions is 2,4,6-trichloro-1,3,5-triazine (TCT), which is generally considered non-toxic. Systems involving this reagent, in combination with either DMSO or DMF, give very good yields in short reaction times with no heating required $[5,6]$. A drawback with these systems is that only two chlorine atoms of TCT are used in the reaction, and therefore atom efficiency is compromised. Another type of chlorination system involves the use of silicon-based chlorinating agents in the presence of Group 13 chlorides (e.g. $\mathrm{InCl}_{3}$ ) as catalysts [7, 8].

Recently reported methodologies concerning bromination and iodination of alcohols are similar in substance to the reports of chlorination processes; some are truly 'green' systems whereas others would not be suitable for scale-up [9-12]. One particular system produces alkyl iodides starting from the corresponding ketone with the use of a ruthenium-based catalyst that is active towards both reduction and halogenation [11]. This system appears to be extremely useful as it can be used for a number of substrates and uses a mild iodinating agent, NaI. However, while crucial to organic synthesis, precious metal catalysis is often not economically feasible on a large scale.

Solvents are required in most organic synthetic processes to produce homogeneous mixtures, provide a means for energy and mass transfer, stabilize transition states, and/or provide a means for separation and purification of products [12]. In the substitution reactions described above, dipolar aprotic solvents (e.g. DMF, DMSO) are often required to dissolve ionic reagents and stabilize intermediates. Ionic liquids are one class of alternative reaction media, which are suitable replacements for such solvents [13]. The field of catalytic reactions performed in ionic liquids has been reviewed extensively and the sheer volume of transformations performed with ionic liquids is outstanding [14-16]. In surveying these reactions, an interesting observation is warranted. Depending on its properties, an ionic liquid can be used as an 'innocent' solvent, a ligand precursor, a co-catalyst, or even the catalyst itself. Ionic liquids have been used as "reagent-solvents" in bromination and iodination of alcohols, where the active nucleophile originates as the anion of the ionic liquid [17-19]. These systems have commonly been used with long-chain alcohols [19], which have previously been problematic to halogenate due to their poor solubility in most solvents. The greenness of these systems is enhanced as the ionic liquid can be regenerated and reused a number of times simply by stirring, with a $\mathrm{NaX}$ salt $(\mathrm{X}=\mathrm{Br}, \mathrm{I})$, at an elevated temperature over a short period of time [19]. This allows a number of reactions to be performed in the same solvent, which is generally unheard of in reactions involving traditional organic solvents.

The research presented in the current article arose from earlier findings in our group [20]. Upon investigating microwave-assisted catalytic dehydrative etherification of benzyl alcohols in ionic liquids, attempts were made to observe the affect of $\mathrm{pH}$ on the reaction. Upon addition of a stoichiometric amount of $\mathrm{NH}_{4} \mathrm{Cl}$ to reactions containing benzyl alcohol as the substrate, the major product obtained was benzyl chloride. As this reaction was peripheral to the original study, no further investigation was performed at that time regarding the conditions and scope of this reaction. As mentioned above, the halogenation of alcohols under relatively mild conditions is an important synthetic transformation and the development of greener methods of performing these reactions is desired. Therefore, it was deemed worthwhile to perform further studies of this halogenation process. The goals of this study commence with the optimization of the chlorination process described above. Attempts were made to observe the affects of different ionic liquids, reaction time and temperature on the chlorination process. Using the optimized conditions as a starting point, reactions were performed on benzyl alcohol using different ammonium salts $\left(\mathrm{NH}_{4} \mathrm{~F}, \mathrm{NH}_{4} \mathrm{Br}\right.$ and $\left.\mathrm{NH}_{4} \mathrm{I}\right)$ to observe if halogenation could be performed using these reagents. A summary of optimization parameters is shown in Fig. 1.

\section{Results and discussion}

Further to our initially reported study [20], we discovered that palladium was not an essential component in the catalytic system for chlorinating benzyl alcohol. Therefore, a number of reaction variables (time, temperature and nature of the ionic liquid) were optimized for the nonmetal catalyzed reaction of benzyl alcohol with $\mathrm{NH}_{4} \mathrm{Cl}$ to yield benzyl chloride. Reaction conditions (MW time and temperature) were optimized for the conversion of benzyl alcohol to benzyl chloride in $\left[\mathrm{Bmim}^{-} \mathrm{PF}_{6}\right.$ (Figs. 2, 3). At $150{ }^{\circ} \mathrm{C}$, after the optimum reaction time of $17 \mathrm{~min}$, an NMR yield of $68 \%$ was obtained. There is a distinct

$$
\begin{aligned}
& \mathrm{ROH} \underset{\mathrm{MW} \text { heating }}{\longrightarrow} \mathrm{RX} \\
& \text { (i) } \mathrm{IL} \text { e.g. [Bmim]PF } \mathrm{F}_{6} \\
& \text { (ii) Temperature }\left(120-180^{\circ} \mathrm{C}\right) \\
& \text { (iii) } \mathrm{Time}(5-35 \text { min) } \\
& \text { (iv) } \mathrm{NH}_{4} \mathrm{X}, \mathrm{X}=\mathrm{Cl}, \mathrm{Br} \text { or I } \\
& \text { (v) } \mathrm{R}=\mathrm{C}_{7} \mathrm{H}_{7}, \mathrm{C}_{4} \mathrm{H}_{9} \text {, etc. }
\end{aligned}
$$

Fig. 1 Parameters to be considered in optimizing catalytic halodehydroxylation of alcohols in ionic liquids 


\section{Reaction Time Study at $150^{\circ} \mathrm{C}$}

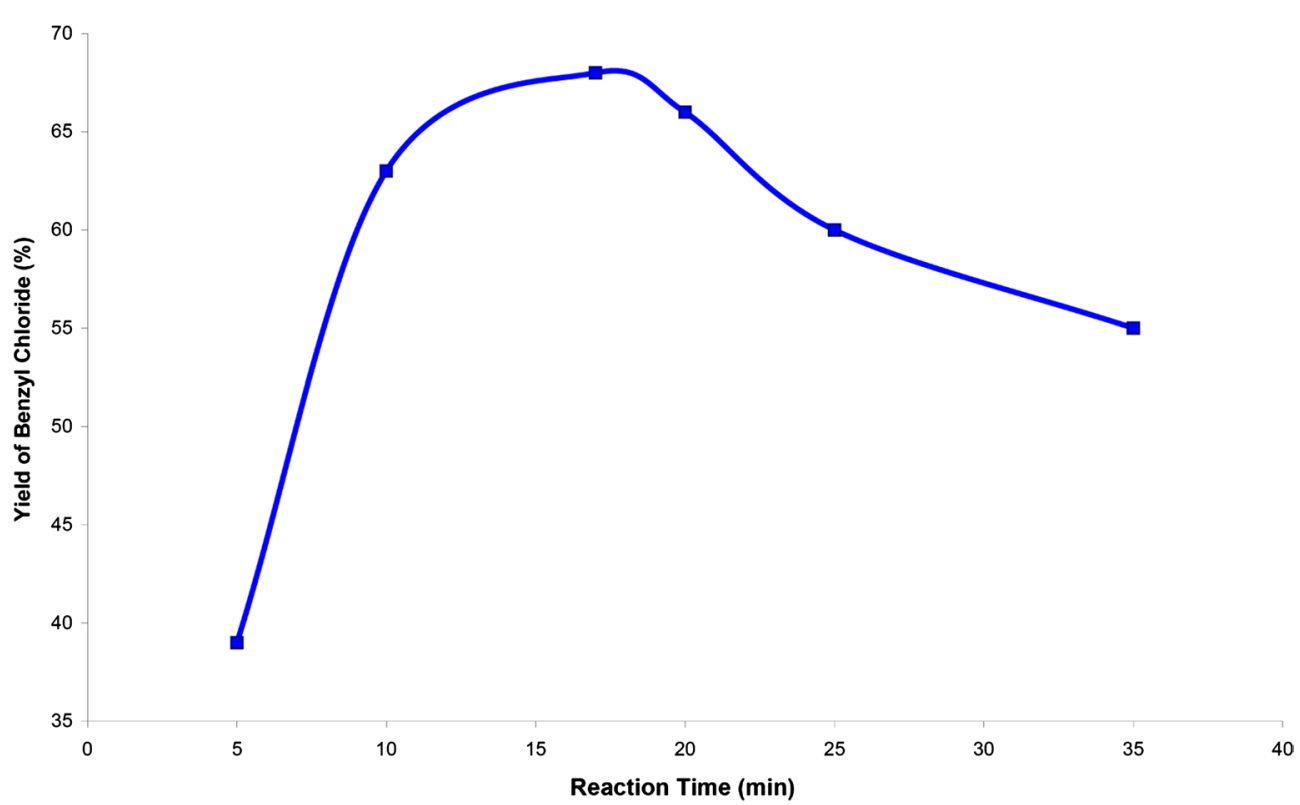

Fig. 2 Reaction time study of the imidazolium catalyzed transformation of benzyl alcohol to benzyl chloride. ([Bmim] $\mathrm{PF}_{6}, 150{ }^{\circ} \mathrm{C}, 17 \mathrm{~min}, 68 \%$ )

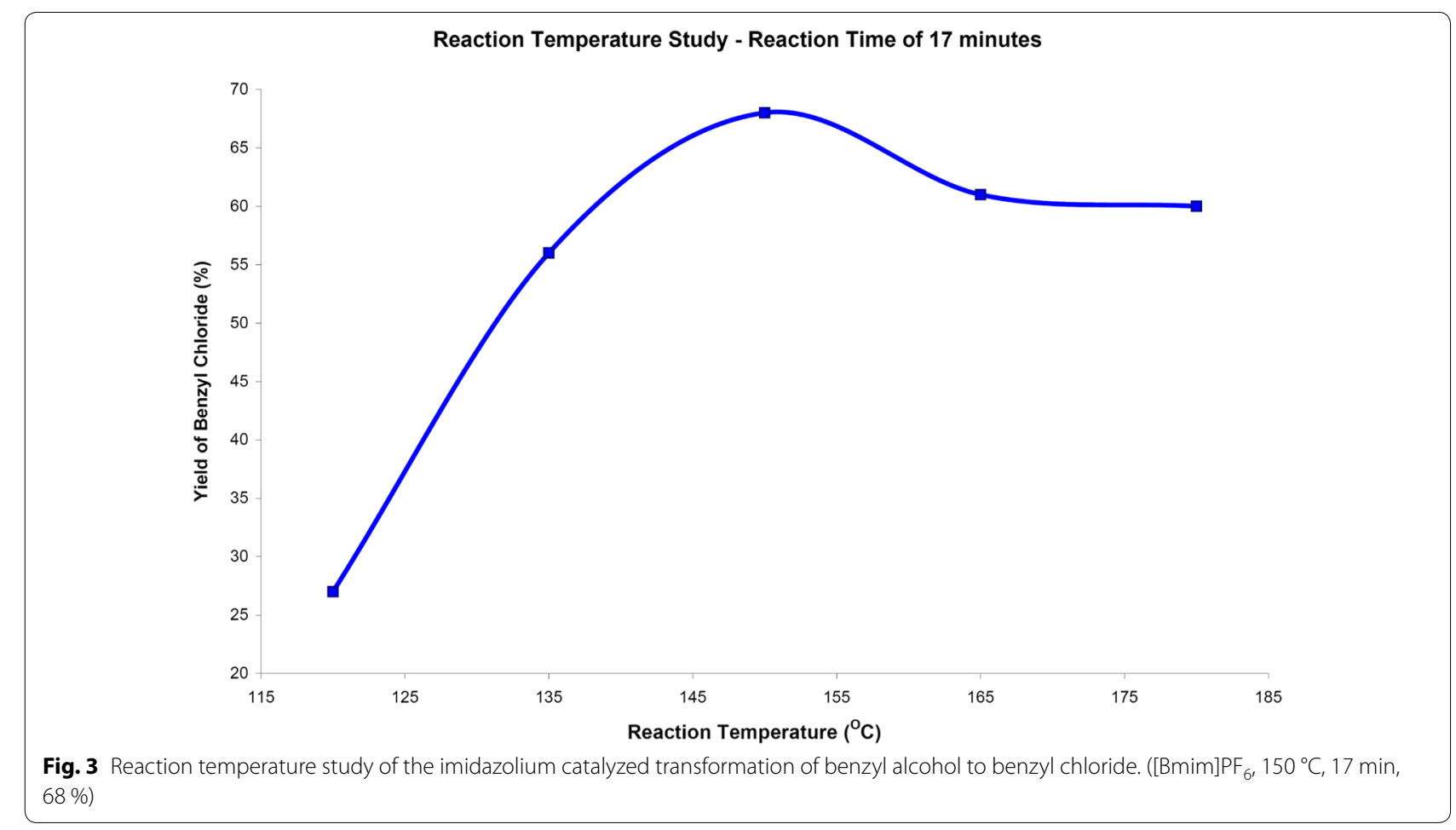

rise to a maximum yield followed by a decrease in yield with longer reaction times. Similar decreases in yield are observed when reactions are performed at higher temperatures. The decrease in yields is a result of byproduct formation such as dibenzylether [20]. Products and unreacted starting materials could be isolated from 
the reaction mixture via vacuum distillation, using apparatus described elsewhere [21]. Purified products could then be isolated via flash chromatography or fractional reduced pressure distillation. Isolated yields (when determined) were not significantly different to those obtained via GC or NMR analyses.

The effect the ionic liquid had on this reaction was investigated while all other variables were kept constant (Table 1). $[\mathrm{Bmim}] \mathrm{PF}_{6}$ was the most suitable solvent for this reaction. The reaction does not occur in hydrophilic ionic liquids, e.g. $\left[\mathrm{Bmim}_{3} \mathrm{BF}_{4}\right.$, nor the conventional hydrophilic solvent DMF. This is not unexpected because dehydration reactions can be enhanced in hydrophobic reaction media. The reaction was also performed in two other hydrophobic ionic liquids: $\left[\mathrm{P}_{66614}\right]$ $\mathrm{DBS}$ and $[\mathrm{BMmim}] \mathrm{PF}_{6}$. This shows that the hydrophobicity of the solvent is not the only requirement and that the cation plays a critical role in the reaction outcome. An interesting inference can be made by comparing the results for $[\mathrm{Bmim}] \mathrm{PF}_{6}$ and $[\mathrm{BMmim}] \mathrm{PF}_{6}(68 \%$ for $[\mathrm{Bmim}] \mathrm{PF}_{6}, 18 \%$ for $\left.[\mathrm{BMmim}] \mathrm{PF}_{6}\right)$. The only difference between these two ionic liquids is the identity of the substituent on the carbon between the nitrogens of the cation (Fig. 4): [Bmim] $\mathrm{PF}_{6}$ contains an acidic proton

Table 1 Reaction screening: effect of reaction medium, halogenating agent and alcohol on dehydroxyhalogenation reactions

\begin{tabular}{|c|c|c|c|}
\hline Alcohol & Solvent & Halide & Yield (\%) \\
\hline $\mathrm{BnOH}$ & {$[\mathrm{Bmim}] \mathrm{PF}_{6}$} & $\mathrm{Cl}$ & $68(65)$ \\
\hline $\mathrm{BnOH}$ & {$\left[\mathrm{BMmim} \mathrm{PF}_{6}\right.$} & $\mathrm{Cl}$ & 18 \\
\hline $\mathrm{BnOH}$ & {$[\mathrm{Bmim}] \mathrm{BF}_{4}$} & $\mathrm{Cl}$ & 0 \\
\hline $\mathrm{BnOH}$ & {$[\mathrm{Bmim}] \mathrm{Cl}$} & $\mathrm{Cl}$ & 0 \\
\hline $\mathrm{BnOH}$ & {$\left[\mathrm{P}_{66614}\right] \mathrm{DBS}$} & $\mathrm{Cl}$ & 5 \\
\hline $\mathrm{BnOH}$ & DMF & $\mathrm{Cl}$ & 0 \\
\hline $\mathrm{BnOH}$ & {$\left[\mathrm{Bmim} \mathrm{PF}_{6}\right.$} & $\mathrm{Br}$ & 50 \\
\hline $\mathrm{BnOH}$ & {$[\mathrm{Bmim}] \mathrm{PF}_{6}$} & 1 & 72 \\
\hline$p-\mathrm{Cl}-\mathrm{BnOH}$ & {$\left[\mathrm{Bmim} \mathrm{PF}_{6}\right.$} & $\mathrm{Cl}$ & $81(77)$ \\
\hline$p-\mathrm{Br}-\mathrm{BnOH}$ & {$\left[\mathrm{Bmim} \mathrm{PF}_{6}\right.$} & $\mathrm{Cl}$ & 65 \\
\hline$p-\mathrm{NO}_{2}-\mathrm{BnOH}$ & {$[\mathrm{Bmim}] \mathrm{PF}_{6}$} & $\mathrm{Cl}$ & 58 \\
\hline$p-\mathrm{CH}_{3}-\mathrm{BnOH}$ & {$[\mathrm{Bmim}] \mathrm{PF}_{6}$} & $\mathrm{Cl}$ & $46^{\mathrm{a}}$ \\
\hline p-OH-BnOH & {$\left[\mathrm{Bmim} \mathrm{PF}_{6}\right.$} & $\mathrm{Cl}$ & 0 \\
\hline$p-\mathrm{CH}_{3} \mathrm{O}-\mathrm{BnOH}$ & {$\left[\mathrm{Bmim} \mathrm{PF}_{6}\right.$} & $\mathrm{Cl}$ & 0 \\
\hline$n-\mathrm{BuOH}$ & {$[\mathrm{Bmim}] \mathrm{PF}_{6}$} & $\mathrm{~F}$ & 0 \\
\hline$n-\mathrm{BuOH}$ & {$\left[\mathrm{Bmim} \mathrm{PF}_{6}\right.$} & $\mathrm{Cl}$ & $51(51)$ \\
\hline$n-\mathrm{BuOH}$ & {$\left[\mathrm{Bmim} \mathrm{PF}_{6}\right.$} & $\mathrm{Br}$ & $65(62)$ \\
\hline$n-\mathrm{BuOH}$ & {$\left[\mathrm{Bmim} \mathrm{PF}_{6}\right.$} & 1 & 82 \\
\hline citronellol & {$\left[\mathrm{Bmim} \mathrm{PF}_{6}\right.$} & $\mathrm{Cl}$ & 50 \\
\hline
\end{tabular}

Conditions (unless otherwise stated): $\mathrm{MW}, 150^{\circ} \mathrm{C}, 15 \mathrm{~min}$. Yield reported obtained via NMR analyses. Value in parentheses is isolated yield

a $\mathrm{T}=125^{\circ} \mathrm{C}$ whereas [BMmim] $\mathrm{PF}_{6}$ bears a methyl group at this position. This observation leads to a hypothesis that the acidic proton of $[\mathrm{Bmim}] \mathrm{PF}_{6}$ is playing a significant role in the production of benzyl chloride. Another interesting conclusion that can be drawn via comparison of the results for $[\mathrm{Bmim}] \mathrm{PF}_{6}$ and $[\mathrm{BMmim}] \mathrm{PF}_{6}$ is that $\mathrm{HF}$, potentially produced via hydrolytic decomposition of the $\mathrm{PF}_{6}{ }^{-}$anion, is not playing a major role in the production of benzyl chloride. Since the hydrolytic instability of $\mathrm{PF}_{6}{ }^{-}$should be independent of the nature of the imidazolium cation. If HF was the active catalyst, it would be expected that both [Bmim] $\mathrm{PF}_{6}$ and [BMmim] $\mathrm{PF}_{6}$ would give very similar results in the chlorination of benzyl chloride. Based on the nature of the imidazolium systems, it was hypothesized that either one of two species was acting as the catalyst: an acidic species or a N-heterocyclic carbine (NHC) generated from the deprotonation of $[\mathrm{Bmim}] \mathrm{PF}_{6}$ (Fig. 5). It was predicted that if the catalyst was acidic, addition of base would hinder the reaction whereas if the catalyst was an NHC, addition of base would improve the yield. Therefore, a reaction was performed where the optimized reaction conditions were used and $\mathrm{KOH}$ (potassium hydroxide) was added. It was found that under these conditions, no benzyl chloride was produced, suggesting that the active catalyst is an acidic species.

\section{Mechanistic and equilibrium considerations}

In this particular system, there are three acidic species that could be proposed as catalysts in this reaction: $\mathrm{NH}_{4}^{+}, \mathrm{HF}$ and the acidic proton of $\left[\mathrm{Bmim}^{+} \mathrm{PF}_{6}\right.$. As [Bmim] Cl could also be used as the chlorinating reagent, one can conclude that $\mathrm{NH}_{4}{ }^{+}$is not the major catalyst as the reaction proceeds even when $\mathrm{NH}_{4}{ }^{+}$is not present. Also, we have already hypothesized that HF is not the catalytic species, as significantly different results were obtained when $[\mathrm{Bmim}] \mathrm{PF}_{6}$ and $[\mathrm{BMmim}] \mathrm{PF}_{6}$ were used, which should produce the same amount of HF if anion decomposition was occurring. Therefore, we propose that the major catalytic species in the presented reaction system is the acidic proton of $[\mathrm{Bmim}] \mathrm{PF}_{6}$. The involvement of this proton is supported by the large decrease in yield (from 68 to $18 \%$ ) when [BMmim] $\mathrm{PF}_{6}$, which does not contain this acidic proton, is used as the solvent instead of $[\mathrm{Bmim}] \mathrm{PF}_{6}$ in the chlorination of benzyl alcohol. The proposal that this acidic proton is involved is based on precedent seen in the literature. Despite having a relatively high $\mathrm{p} K_{\mathrm{a}}$ of 21-24 [22], this proton has been observed to participate in a number of interactions. In one particular case, where Michael addition reactions were studied, results obtained suggested that the acidic 


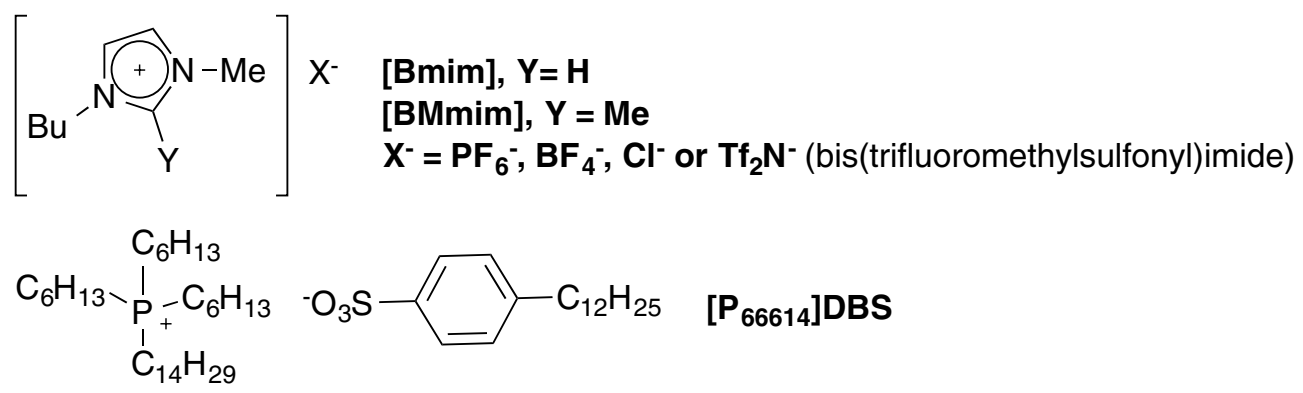

Fig. 4 lonic liquids screened for suitability in the conversion of benzyl alcohol to benzyl chloride

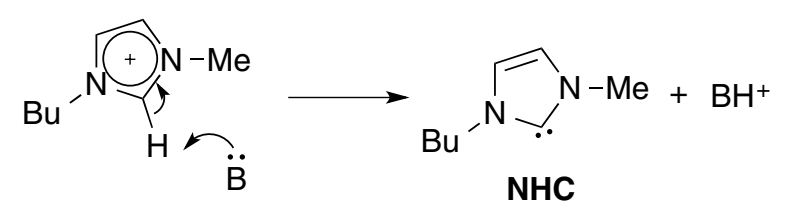

Fig. 5 Formation of $\mathrm{NHC}$ from $[\mathrm{Bmim}]^{+}$in the presence of base (B)

proton of $[\mathrm{Bmim}] \mathrm{PF}_{6}$ formed a hydrogen bond with a basic catalyst, resulting in a decrease of activity [23].

In nucleophilic substitution reactions, there are two general mechanisms: $\mathrm{S}_{\mathrm{N}} 1$ and $\mathrm{S}_{\mathrm{N}} 2$. For $\mathrm{S}_{\mathrm{N}} 1$ reactions, the leaving group (in this case - $\mathrm{OH}$ ) is ejected, forming a carbocation intermediate, which is attacked by the nucleophile. In general, $\mathrm{S}_{\mathrm{N}} 1$ reactions are favored by good leaving groups and highly branched systems that can form a stable carbocation intermediate (e.g. $3^{\circ}$ alkyl reagents). For $\mathrm{S}_{\mathrm{N}} 2$ reactions, an incoming nucleophile attacks from the backside of the substrate and ejects the leaving group. $\mathrm{S}_{\mathrm{N}} 2$ reactions are favored by good nucleophiles and unhindered $\mathrm{C}-\mathrm{X}$ bonds such as primary alcohols. In order to determine whether an $\mathrm{S}_{\mathrm{N}} 1$ or $\mathrm{S}_{\mathrm{N}} 2$ process dominates, competition studies were performed. Reaction of one equiv. $\mathrm{NH}_{4} \mathrm{Cl}$ with one equiv. of both benzyl alcohol and 1-phenyl ethanol was performed. This afforded benzyl chloride in yields identical to those performed in the absence of 1-phenyl ethanol. Under the reaction conditions explored, there was no evidence of 1-phenyl ethanol reacting. This lack of reactivity suggests that the reaction does not proceed in an $\mathrm{S}_{\mathrm{N}} 1$ fashion, as the more stable carbocation should afford higher yields of 1-phenyl-chloroethane than benzyl chloride.

The proposed mechanism for the conversion of benzyl alcohol to benzyl chloride for the reaction system used in this study is presented in Fig. 6. As described in the background section, hydroxyl moieties are very poor leaving groups and usually require activation of some sort in order to be displaced by a nucleophile. This is usually done by protonating the oxygen of the hydroxyl group

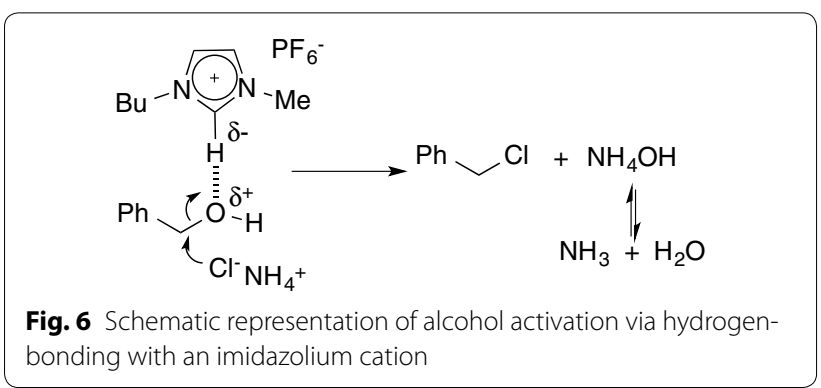

with acid, producing an excellent leaving group. In the proposed mechanism (Fig. 6), we suggest that the activation of the alcohol occurs through hydrogen-bonding of the acidic proton of the imidazolium cation with the oxygen of the alcohol, causing the alcohol to bear a slight positive charge. This causes the alcohol to be activated and subsequently displaced by the chloride nucleophile in a $\mathrm{S}_{\mathrm{N}} 2$ fashion.

The examination of this proposed mechanism allows for rationalization of some of the previously described results. For the reaction performed in the phosphonium based ionic liquid, $\left[\mathrm{P}_{66614}\right] \mathrm{DBS}$, a very low yield of product was observed. Since this ionic liquid does not contain an acidic site similar to [Bmim] $\mathrm{PF}_{6}$, without this acidic site, activation of the alcohol could not be achieved, leading to very poor conversions.

In our previous studies involving dehydration reactions of benzyl alcohols [20,21], equilibria between the alcohol and product were observed. In the studies herein varying quantities of dibenzyl ether co-product were noted. This led to problems in obtaining better yields of benzyl chloride with longer reaction times. To investigate the role of dibenzyl ether in these halogenation processes, a reaction was attempted, using the optimized conditions $\left(17 \mathrm{~min}, 150{ }^{\circ} \mathrm{C}\right.$, $\left.[\mathrm{Bmim}] \mathrm{PF}_{6}\right)$ where dibenzyl ether and a molar equivalent of water was reacted in the presence of $\mathrm{NH}_{4} \mathrm{Cl}$ instead of benzyl alcohol (Fig. 7). From this reaction, it was found that after the reaction, 
$2 \mathrm{BnCl}+2 \mathrm{NH}_{3}+\mathrm{H}_{2} \mathrm{O} \rightleftharpoons \mathrm{BnOBn}+2 \mathrm{NH}_{4} \mathrm{Cl}$

Fig. 7 Observed equilibrium between benzyl chloride and dibenzyl ether in the presence of water and ammonium chloride

both benzyl chloride and dibenzyl ether were present. This result indicates that an equilibrium does indeed exist between the two compounds, and explains the observed time dependence on the yield of benzyl chloride. As the reaction proceeds and water is produced, the yield of benzyl chloride decreases as the yield of dibenzyl ether increases.

Since benzyl chloride is the desired product in the reactions that have been presented thus far, it would be desirable to control the selectivity of these reactions and improve the yield of benzyl chloride. In the dehydration reaction of benzyl alcohol to produce diben$\mathrm{zyl}$ ether, water is produced as a by-product. However, based on the mechanism proposed in Fig. 6 for the formation of benzyl chloride from benzyl alcohol, hydroxide is formally produced as a by-product. While the majority of the hydroxide produced may undergo rapid proton transfer with $\mathrm{NH}_{4}{ }^{+}$to produce $\mathrm{H}_{2} \mathrm{O}$, the highly ionic nature of the solvent may allow a substantial amount of the hydroxide to exist as a charged species in solution. Based on this argument, we hypothesized that the presence of a small amount of water added to the reaction mixture would hinder the production of dibenzyl ether more than it would suppress the production of benzyl chloride. In order to investigate this prediction, the best yielding reaction presented above (68\% yield of benzyl chloride) was attempted in the presence of a half molar equivalent of water with respect to benzyl alcohol to see what effect this had on the yield. It was found that this reaction yielded $75 \%$ benzyl chloride, improving the yield slightly from what was previously observed. This result is in agreement with the rationale presented above. However, the amount of water added needs to be carefully controlled. When three molar equivalents of $\mathrm{H}_{2} \mathrm{O}$ are added, only a $39 \%$ yield of benzyl chloride was obtained. Nevertheless, these findings show that dehydroxylation reactions need to be explored carefully in terms of the water content of the reaction medium and methods for removing water to enhance yields.

In summary, it has been discovered that an equilibrium exists between benzyl chloride and dibenzyl ether in the reactions that have been studied and that some control over this equilibrium can be acquired with the addition of a small amount of water to the reaction mixture. However, in order to improve the yield of benzyl chloride, it is necessary to gain further control of the production of dibenzyl ether, either through an additive or catalytic species.

\section{Extension of this reaction to bromide, iodide and other alcohols}

Using the optimized reaction conditions for the conversion of benzyl alcohol to benzyl chloride, attempts were made to halogenate benzyl alcohol with $\mathrm{NH}_{4} \mathrm{Br}$ and $\mathrm{NH}_{4} \mathrm{I}$. The results for these reactions are summarized in Table 1. The yield of benzyl iodide is quite similar to the yield of benzyl chloride under the same conditions (72 versus $68 \%$ ), but the yield of benzyl bromide (50\%) is significantly lower. Based on the proposed mechanism, it would be expected that the best nucleophile, iodide, would give the best yield followed by bromide and chloride. Since dibenzyl ether was also observed as a product in the reactions involving $\mathrm{NH}_{4} \mathrm{Br}$ and $\mathrm{NH}_{4} \mathrm{I}$, it is expected that similar equilibria exist in these halogenation reactions, as was the case for the chlorination reaction. Therefore, these yields are not completely comparable as the time and temperature dependence of the bromination and iodination reactions have not been fully investigated. Fluorination of benzyl alcohol was attempted with $\mathrm{NH}_{4} \mathrm{~F}$ using a number of reaction conditions $\left(80-180{ }^{\circ} \mathrm{C}\right.$, 15-40 $\mathrm{min}$ ). For all of these reactions, it was not possible to identify any fluorinated product, although all of the starting material had reacted, in most cases. This result is not surprising based on the properties of the fluoride anion, which is a poor nucleophile but extremely basic and rarely reacts neatly in nucleophilic displacement reactions.

Using the optimized conditions for the chlorination of benzyl alcohol, attempts were made to halogenate a variety of other alcohols. These included substituted benzyl alcohols, both functionalized and non-functionalized aliphatic alcohols, and a secondary alcohol. The results of these studies are discussed below.

The first group of alcohols that were studied were parasubstituted benzyl alcohols, to investigate the effect substituents had on the yield of the chlorination reactions. The results obtained are shown in Table 1. Products were identified via ${ }^{1} \mathrm{H}$ NMR spectra of known compounds in combination with GC-MS analysis of the reaction mixtures. Isolated yields for some products are also reported. From these results, a number of interesting observations are made. Firstly, it is obvious that the yield of the chlorinated product is very much dependent on the substituent at the para-position of the aromatic ring. This is opposite to what was observed in the etherification reactions of benzyl alcohols, using a similar reaction system [20], where it was found that the substituent at the paraposition had little effect on the yield of the etherification product. For the chlorination reactions, in comparison to when $\mathrm{Y}=\mathrm{H}$, it is seen that when $\mathrm{Y}=\mathrm{Cl}$, the yield of the chlorinated benzyl alcohol is improved whereas when $\mathrm{Y}=\mathrm{Br}, \mathrm{NO}_{2}$ or $\mathrm{CH}_{3}$, the yield decreases. These trends 
can be rationalized using a number of arguments. When $\mathrm{Y}=\mathrm{CH}_{3}$ and the reaction is attempted at $150{ }^{\circ} \mathrm{C}$, a large number of by-products were observed from the GC-MS analysis making calculation of the yield of the chlorinated product difficult. However, by lowering the temperature to $125^{\circ} \mathrm{C}$, better control of the reaction was obtained and a $46 \%$ yield of 4-methylbenzyl chloride could be determined. This suggests that at higher temperatures the chlorinated product is generated but reacts further to give the observed side-products (incl. aryl-substituted methanes). This can be rationalized by considering that with an electron-donating group in the para-position, such as $\mathrm{CH}_{3}$, the benzylic carbocation that could be generated in the reaction mixture would react to give high mass by-products (e.g. di( $p$-tolyl)methane). For $\mathrm{Y}=\mathrm{Br}$ and $\mathrm{Cl}$, one would expect similar yields. This is not the case as when $\mathrm{Y}=\mathrm{Br}$, the yield obtained was $65 \%$ whereas when $\mathrm{Y}=\mathrm{Cl}$ the yield was $81 \%$. In the case when $\mathrm{Y}=\mathrm{Br}$ or $\mathrm{Cl}$, the corresponding etherification product is also obtained in a significant amount. As was explored to a certain extent above (Fig. 7), an equilibrium exists between the chlorinated product and the etherification product that is highly dependent upon reaction time. It is predicted that this equilibrium is also dependent upon electronic properties of the aromatic ring system, which is based upon the nature of the substituents. Therefore, it is possible that for $\mathrm{Y}=\mathrm{Br}$ and $\mathrm{Cl}$, the reaction conditions used did not optimize the yield of the chlorinated product. For the reaction involving 4-nitrobenzyl alcohol $\left(\mathrm{Y}=\mathrm{NO}_{2}\right)$, a number of interesting observations were made. From the GC-MS analysis, it is shown that the only product obtained was 4-nitrobenzyl chloride, along with unreacted starting material. This was the only benzyl alcohol substrate that did not produce any ether by-product, which is consistent with the results obtained previously [20]. These observations suggest that the nitro group extensively modulates the reactivity of the alcohol and may give rise to some information regarding the mechanism of the etherification reactions. In comparison to the reaction of benzyl alcohol under identical conditions, it is observed that the nitro-substituted benzyl alcohol produces a slightly lower yield of the desired product (58 versus $68 \%$ ). This result is not surprising and can be rationalized using the finer details of the proposed mechanism of these reactions (Fig. 6). In this mechanism, a slight positive charge is generated, suggesting that a build-up of positive charge in the transition state will also be observed. In the presence of an electron-withdrawing group, such as $\mathrm{NO}_{2}$, this positively charged species would be destabilized, increasing the energy of the transition state and slowing the transformation of the alcohol to the chloride. No yield of chlorinated product was obtained for $\mathrm{Y}=\mathrm{OH}$ and $\mathrm{OCH}_{3}$. Upon attempting to analyze the products of these reactions, it was found that the reaction mixtures were uncharacteristically viscous and a product had formed that did not dissolve in organic solvents (ether, acetone and hydrocarbons). These observations suggested that some sort of polymeric product formed in these reactions, as proposed in Fig. 8. This also implies that the methoxy group of 4-methoxybenzyl alcohol undergoes hydrolysis under these reaction conditions, producing the phenoxide which could further react in a similar way as 4-hydroxybenzyl alcohol to give a polymeric product. These results show that unfortunately this method is not applicable to all types of benzylic alcohols.

In order to investigate substrate scope, chlorination was attempted using 2-phenylethanol as the substrate. Using the optimum reaction conditions for chlorination of benzyl alcohol, 2-phenylethyl chloride was obtained in $60 \%$ yield. From the GC-MS analysis, it was observed that only a very small amount of the etherification product was obtained and that no other by-products were produced. This result suggests that benzyl alcohols are much more reactive in this system, leading to unwanted side-products. Interestingly, the chlorination of this substrate was studied with an indium-catalysed system that used chlorodimethylsilane as the chlorinating agent, and although this system was successful in chlorinating a wide range of alcohols, no yield was obtained for this particular substrate [8]. This result was rationalized with the prediction that a carbocation intermediate was being formed and since 2-phenylethanol is a primary alcohol, a primary carbocation would have to be formed, which is unflavored [8]. The fact that the system presented herein is effective in the transformation of a primary alcohol to a primary chloride further supports the $\mathrm{S}_{\mathrm{N}} 2$-like mechanism described above.

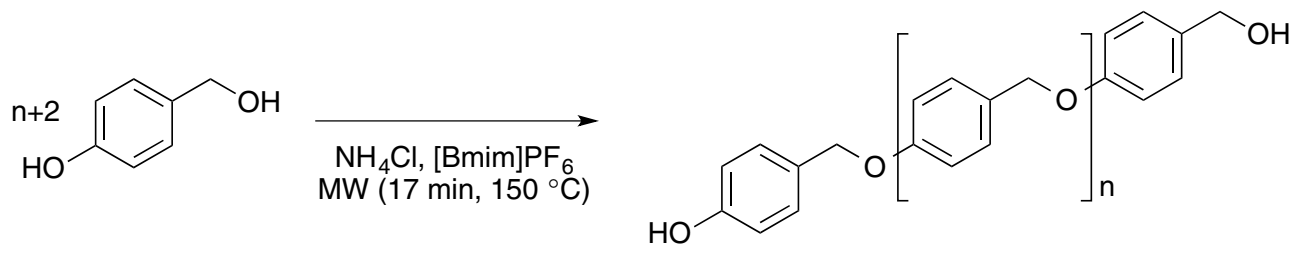

Fig. 8 Postulated reaction from attempted chlorination of 4-hydroxybenzyl alcohol 
With the discovery that this chlorination system was effective in the chlorination of 2-phenylethanol, investigations were performed to see if this system could be expanded to simple aliphatic alcohols. Since it is known that the $[\mathrm{Bmim}]^{+}$cation interacts favorably with aromatic systems [24], the halogenation of 1-butanol (Table 1) was investigated to see if the presence of the aromatic ring was required for the halogenation to occur. The yield of the butyl halide increases with increasing nucleophilicity of the anion reacted (best yield obtained with $\mathrm{NH}_{4} \mathrm{I}$ ). This is the trend expected when studying substitution reactions that follow $\mathrm{S}_{\mathrm{N}} 2$ mechanisms. This mechanism is also supported by the observation that no 2-butyl halides or rearrangement products were obtained based on analysis of the ${ }^{1} \mathrm{H}$ NMR spectra for the reactions involving 1-butanol. One would expect that if a $S_{N} 1$ mechanism existed in these reactions, rearrangement products would be observed, as is the case with the indium-catalysed system [8]. As well, it should be noted that no ether by-products were observed in these reactions, suggesting that etherification under these reaction conditions is limited primarily to compounds containing aromatic groups. Given the selectivity of these reactions, ionic liquid recycling studies were performed for the n-butanol to 1 -chlorobutane process. The reaction was performed $\left(150{ }^{\circ} \mathrm{C}, 17 \mathrm{~min}\right)$ and after completion, the product could be isolated via distillation. The diluted ionic liquid phase containing some unreacted alcohol was dried over anhydrous sodium sulfate, decanted, concentrated and then successfully re-used for the same reaction four times. Yields obtained were $50-57 \%$ (based on the amount of alcohol added before each reaction).

Although it was observed that halogenation could be performed on aliphatic alcohols, the effect of functional groups on the halogenation process had not yet been determined. For this study, the renewable alcohol citronellol was chosen (Table 1) as it contains a double bond that could be reactive under the acidic conditions contained in this chlorination system. As before, the optimized conditions obtained from the study of benzyl alcohol were used. From the GC-MS analysis of this reaction, it was found that the desired chlorinated product was obtained as thea major product. A number of other by-products were obtained in small amounts such as the terminal alkene generated from an elimination reaction involving either the hydroxyl of the starting material or the generated chloride. This suggests that for the most part, the double bond functionality of citronellol is insensitive to the reaction conditions and similar compounds could be halogenated. However, if more acidsensitive functional groups were present in the starting material, such as terminal alkenes or esters, it is predicted these groups would not be able to withstand the conditions of this halogenation system.

Based on the $\mathrm{S}_{\mathrm{N}} 2$-like mechanism proposed, it would be expected that the halogenation of secondary and tertiary alcohols using this system would be more difficult then benzylic and primary alcohols due to steric hindrance. This hypothesis was investigated by analyzing the reaction of 1-indanol (Fig. 9), a secondary alcohol, under the optimum conditions for halogenation of benzyl alcohol. Analysis of the reaction by ${ }^{1} \mathrm{H}$ NMR and GC-MS led to a very interesting discovery. From the GC trace, only one major product was observed. The mass spectrum of this compound shows that the molecular ion has $\mathrm{m} / \mathrm{z}$ 232.2, which corresponds to the alkene-bound dimer shown in Fig. 9. This type of product had not been seen in any of the other previous halogenation reactions performed and is highly unusual. The product was isolated (63\% yield) via flash chromatography and characterized via NMR spectroscopy, and high resolution mass spectrometry, which confirmed the identity of the product as spectra were in agreement with literature data [25]. Further investigation of this reaction would be worthwhile to see if it could be expanded for use with other secondary alcohols. If expansion were possible, this system could pose as an alternative to the McMurry reaction.

Overall, it has been shown that $\mathrm{NH}_{4} \mathrm{X}(\mathrm{X}=\mathrm{Cl}, \mathrm{Br}, \mathrm{I})$ in $[\mathrm{Bmim}] \mathrm{PF}_{6}$ under microwave irradiation can be used to halogenate a number of alcohols. Fluorination was not possible under the conditions explored. With respect to para-substituted benzyl alcohols, the halogenation process is highly dependent upon the nature of the substituent. For aliphatic alcohols, the results obtained support a $\mathrm{S}_{\mathrm{N}} 2$ mechanism, as the yield is directly correlated to the nucleophilicity of the anion used and no rearrangement products are observed. It has also been shown that it is possible to chlorinate unsaturated aliphatic alcohols. However, it is predicted that this system would not be suitable to halogenate alcohols containing acid-sensitive functional groups. For 1-indanol, a secondary alcohol, an unusual coupled product was obtained upon attempting chlorination. Therefore, further investigation into the reactivity of secondary and tertiary alcohols in this reaction system may be warranted.

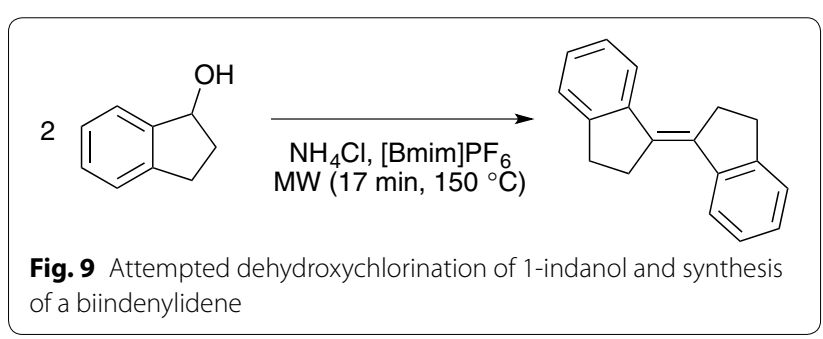




\section{Experimental}

\section{Chemicals and instrumentation}

$[\mathrm{Bmim}] \mathrm{Cl}, \quad[\mathrm{Bmim}] \mathrm{Br}, \quad[\mathrm{Bmim}] \mathrm{Tf}_{2} \mathrm{~N}, \quad\left[\mathrm{Bmim}^{\mathrm{B}}\right] \mathrm{BF}_{4}$ and $[\mathrm{Bmim}] \mathrm{PF}_{6}$ were prepared according to literature procedures [26-28]. [BMmim] $\mathrm{PF}_{6}$ (BMmim, 1-butyl-2,3-dimethylimidazolium) was purchased from Alfa Aesar, while $\left[\mathrm{P}_{66614}\right] \mathrm{DBS}$ was received as a gift from Cytec Inc. Benzyl alcohol was distilled under vacuum and stored under a blanket of nitrogen in contact with 4 A activated molecular sieves. Butanol was distilled from sodium hydroxide $(\mathrm{NaOH})$ and stored, with $4 \AA$ molecular sieves, under nitrogen. All other reagents were purchased from either Alfa Aesar or Sigma Aldrich and used without further purification.

A Biotage microwave reactor was used to run the experiments under microwave (MW) irradiation. The 'very high' absorption level setting was used each time to ensure controlled heating of the reaction. ${ }^{1} \mathrm{H}$ NMR spectra were acquired on a Bruker AVANCE $500 \mathrm{MHz}$ spectrometer with TMS as the internal standard. GC-MS spectra were recorded on an Agilent 7890A GC system coupled with an Agilent 5975C MS detector that was equipped with a capillary column DB-5 (column length: $30.0 \mathrm{~m}$ and column diameter: $0.25 \mathrm{~mm}$ ). All ${ }^{1} \mathrm{H}$ NMR experiments were performed with acetone- $\mathrm{d}_{6}$ as the solvent, except when $\left[\mathrm{P}_{66614}\right]$ DBS was used, in which case the ${ }^{1} \mathrm{H}$ NMR experiments were performed in chloroform-d. In all cases, samples were injected into the GCMS instrument with use of diethyl ether as the solvent. All yields were determined by ${ }^{1} \mathrm{H}$ NMR with use of acetophenone as the internal standard and reported in reference to the limiting reagent. Products could be isolated via vacuum distillation or flash chromatography.

\section{General procedure for dehydroxyhalogenation reactions}

Ammonium salt $(1.10 \mathrm{mmol})$, alcohol $(1.10 \mathrm{mmol})$, and ionic liquid $(0.50 \mathrm{~g})$ were placed in a $2 \mathrm{~mL}$ microwave vial equipped with a stir-bar. The vial was sealed under nitrogen and the reaction mixture was stirred at room temperature for $15 \mathrm{~min}$ to ensure homogeneity. The reaction was then subjected to microwave irradiation for a set period of time at a pre-selected temperature, both of which are noted in the results section above. Upon cooling, a sample of known mass was taken from the reaction mixture, added to a known amount of acetophenone (internal standard) and dissolved in acetone- $\mathrm{d}_{6}$ to be analyzed by ${ }^{1} \mathrm{H}$ NMR. For analysis by GC-MS, a $0.1 \mathrm{~g}$ sample of the reaction mixture was extracted with $2.5 \mathrm{~mL}$ of dry diethyl ether. For product isolation, the ionic liquid was extracted with $3 \times 5 \mathrm{~mL}$ diethyl ether. The combined extracts were concentrated and flash chromatography was performed using a
Biotage Isolera system to separate any unreacted alcohol and ether by-products from the desired halogenated product.

Ionic liquid recycling was performed as follows. Ammonium chloride $(4.40 \mathrm{mmol}), \mathrm{n}$-butanol $(4.40 \mathrm{mmol})$, and $[\mathrm{Bmim}] \mathrm{PF}_{6}(2.00 \mathrm{~g})$ were placed in a microwave vial. The mixture was microwave-heated to $150{ }^{\circ} \mathrm{C}$ for $17 \mathrm{~min}$ whilst stirring. Upon cooling, the vial was transferred to a distillation apparatus and the chlorobutane isolated [21]. The ionic liquid phase was then treated as follows prior to re-use. The contents of the microwave vial were dissolved in anhydrous acetone $(10 \mathrm{~mL})$ and dried over anhydrous sodium sulfate. The mixture was decanted and the acetone removed under vacuum. The resulting ionic liquid was then re-used. Yields of chlorobutane were 51, $57,56,54$, and $52 \%$ (based on the amount of alcohol added before each reaction).

\section{Conclusions}

Further investigation of catalytic chlorodehydroxylation of benzyl alcohol in ionic liquids led to a number of interesting observations. Firstly, although it was initially thought that this transformation was palladium-catalysed [20], it has been proven that this transformation is acidcatalysed and that the presence of palladium has a negative effect on the yield of the desired product. As well, when benzyl alcohols, with the exception of 4-nitrobenzyl alcohol, are employed in this reaction system, an equilibrium exists between the chlorinated product and the corresponding ether that is highly dependent upon reaction temperature, time and substituent. Furthermore, based on experimentally obtained data, it appears that the dehydroxyhalogenation reaction proceeds through a $\mathrm{S}_{\mathrm{N}} 2$ mechanism and that the acidic proton of [Bmim] $\mathrm{PF}_{6}$ plays a crucial role in this process. It has also been shown that this reaction system can be expanded for use with other ammonium halide salts, with the exception of $\mathrm{NH}_{4} \mathrm{~F}$, and both functionalized and non-functionalized primary alcohols. However, when 1-indanol, a secondary alcohol, was used, no chlorinated product was observed and an interesting biindenylidene product was formed in moderate yields. It is known that biindenylidenes have interesting photochromic properties [1, 29-31]. Overall, $\mathrm{NH}_{4} \mathrm{X}(\mathrm{X}=\mathrm{Cl}, \mathrm{Br}, \mathrm{I})$ in $[\mathrm{Bmim}] \mathrm{PF}_{6}$ under microwave irradiation is a safe, green method of transforming several alcohols into the corresponding halides.

\section{Abbreviations}

[Bmim]: 1-butyl-3-methylimidazolium; [BMmim]: 1-butyl-2,3-dimethylimidazolium; $\left[\mathrm{P}_{66614}\right]$ : trihexyl-tetradecylphosphonium; DBS: dodecylbenzenesulfonate; $\mathrm{PPh}_{3}$ : triphenylphosphine; TCT: 2,4,6-trichloro-1,3,5-triazine; DMF: $N, N^{\prime}$ dimethylformamide; DMSO: dimethylsulfoxide; GC-MS: gas chromatographymass spectrometry; OAc: acetate; TMS: tetramethylsilane; MW: microwave. 


\section{Authors' contributions}

CP performed the majority ( $80 \%$ ) of reactions and analyses with the remaining portion performed by HK (20\%). FK conceived of the study, and participated in its design and coordination and wrote the manuscript. All authors read and approved the final manuscript.

\section{Acknowledgements}

We thank Memorial University, the Natural Sciences and Engineering Research Council of Canada (NSERC), Canada Foundation for Innovation (CFI) and Research and Development Corporation (RDC) of Newfoundland and Labrador for funding.

Contribution for special issue on "Green and sustainable solvents".

\section{Competing interests}

The authors declare that they have no competing interests.

Received: 26 May 2015 Accepted: 2 October 2015

Published online: 13 October 2015

\section{References}

1. Constable DJC, Dunn PJ, Hayler JD, Humphrey GR, Leazer JL Jr, Linderman RJ, Lorenz K, Manley J, Pearlman BA, Wells A, Zaks A, Zhang TY (2007) Key green chemistry research areas-a perspective from pharmaceutical manufacturers. Green Chem 9:411-420. doi:10.1039/b703488c

2. An J, Denton RM, Lambert TH, Nacsa ED (2014) The development of cataIytic nucleophilic substitution reactions: challenges, progress and future directions. Org Biomol Chem 12:2993-3003. doi:10.1039/c4ob00032c

3. Pluempanupat W, Chantarasriwong O, Taboonpong P, Jang DO, Chavasiri

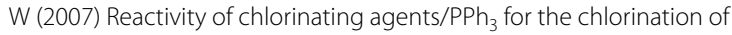
alcohols and carboxylic acids: a comparative study. Tetrahedron Lett 48:223-226. doi:10.1016/j.tetlet.2006.11.060

4. Pluempanupat W, Chavasiri W (2006) An efficient method for chlorination of alcohols using $\mathrm{PPh}_{3} / \mathrm{Cl}_{3} \mathrm{CCONH}_{2}$. Tetrahedron Lett 47:6821-6823. doi:10.1016/j.tetlet.2006.07.060

5. De LL, Giacomelli G, Porcheddu A (2002) An efficient route to alkyl chlorides from alcohols using the complex TCT/DMF. Org Lett 4:553-555

6. Sun L, Peng G, Niu H, Wang Q, Li C (2008) A highly chemoselective and rapid chlorination of benzyl alcohols under neutral conditions. Synthesis 3919-3924. doi:10.1055/s-0028-1083243

7. Yasuda M, Shimizu K, Yamasaki S, Baba A (2008) Direct chlorination of alcohols with chlorodimethylsilane catalyzed by a gallium trichloride/ tartrate system under neutral conditions. Org Biomol Chem 6:2790-2795. doi:10.1039/b804589e

8. Yasuda M, Yamasaki S, Onishi Y, Baba A (2004) Indium-catalyzed direct chlorination of alcohols using chlorodimethylsilane-benzil as a selective and mild system. J Am Chem Soc 126:7186-7187. doi:10.1021/ja048688t

9. Firouzabadi $\mathrm{H}$, Iranpoor N, Ebrahimzadeh $\mathrm{F}$ (2006) Facile conversion of alcohols into their bromides and iodides by N-bromo- and N-iodosaccharins/triphenylphosphine under neutral conditions. Tetrahedron Lett 47:1771-1775. doi:10.1016/j.tetlet.2006.01.033

10. Tongkate P, Pluempanupat W, Chavasiri W (2008) Hexabromoacetone and ethyl tribromoacetate: a highly efficient reagent for bromination of alcohol. Tetrahedron Lett 49:1146-1148. doi:10.1016/j.tetlet.2007.12.061

11. Bhor MD, Panda AG, Nandurkar NS, Bhanage BM (2008) Synthesis of alkyl iodides/nitriles from carbonyl compounds using novel ruthenium tris(2,2,6,6-tetramethyl-3,5-heptanedionate) as catalyst. Tetrahedron Lett 49:6475-6479. doi:10.1016/j.tetlet.2008.09.002

12. (12) Kerton FM (2013) Chapter 1: Introduction. In: Kerton FM, Marriott $\mathrm{R}$ (eds) Alternative solvents for green chemistry. The Royal Society of Chemistry: Cambridge, UK, p 1-30
13. Freemantle M (2009) An introduction to ionic liquids. The Royal Society of Chemistry, Cambridge

14. Dupont J, de Souza RF, Suarez PAZ (2002) Ionic liquid (molten salt) phase organometallic catalysis. Chem Rev 102:3667-3692. doi:10.1021/ cr010338r

15. Welton T (2004) lonic liquids in catalysis. Coord Chem Rev 248:24592477. doi:10.1016/j.ccr.2004.04.015

16. Hallett JP, Welton T (2011) Room-temperature ionic liquids: solvents for synthesis and catalysis. 2. Chem Rev 111:3508-3576. doi:10.1021/ cr1003248

17. Leadbeater NE, Torenius HM, Tye H (2003) lonic liquids as reagents and solvents in conjunction with microwave heating: rapid synthesis of alkyl halides from alcohols and nitriles from aryl halides. Tetrahedron 59:2253-2258. doi:10.1016/S0040-4020(03)00214-X

18. Nguyen H-P, Kirilov P, Matondo H, Baboulene M (2004) The reusable couple "PTSA/1-alkyl-3-methylimidazolium ionic liquids": excellent reagentscatalysts for halogenation of fatty diols. J Mol Catal A Chem 218:41-45. doi:10.1016/j.molcata.2004.04.004

19. Nguyen H-P, Matondo H, Baboulene M (2003) lonic liquids as catalytic "green" reactants and solvents for nucleophilic conversion of fatty alcohols to alkyl halides. Green Chem 5:303-305. doi:10.1039/b303892k

20. Kalviri HA, Petten CF, Kerton FM (2009) Catalytic dehydrative etherification and chlorination of benzyl alcohols in ionic liquids, Chem Commun 5171-5173. doi:10.1039/b909866f

21. Kalviri HA, Kerton FM (2011) Dehydration of benzyl alcohols in phosphonium ionic liquids: synthesis of ethers and alkenes. Adv Synth Catal 353:3178-3186. doi:10.1002/adsc.201100445

22. Sowmiah S, Srinivasadesikan V, Tseng M-C, Chu Y-H (2009) On the chemical stabilities of ionic liquids. Molecules 14:3780-3813

23. Mečiarová M, Cigáň M, Toma Š, Gáplovský A (2008) Kinetic study of michael addition catalyzed by n-methylimidazole in ionic liquids: residual n-methylimidazole in ionic liquids as a strong base. Eur J Org Chem 2008:4408-4411. doi:10.1002/ejoc.200800621

24. Dupont J, Suarez PAZ, De Souza RF, Burrow RA, Kintzinger J-P (2000) $\mathrm{CH}-$ interactions in 1-n-butyl-3-methylimidazolium tetraphenylborate molten salt: solid and solution structures. Chem A Euro J 6:2377-2381 doi:10.1002/1521-3765(20000703)6:13<2377:AID-CHEM2377>3.0.CO;2-L

25. Spiteller $P$, Jovanovic J, Spiteller M (2003) NMR analysis of biindenylidenes. Magn Reson Chem 41:475-477. doi:10.1002/mrc.1165

26. Suarez PAZ, Dullius JEL, Einloft S, De Souza RF, Dupont J (1996) The use of new ionic liquids in two-phase catalytic hydrogenation reaction by rhodium complexes. Polyhedron 15:1217-1219. doi:10.1016/0277-5387(95)00365-7

27. Huddleston JG, Visser AE, Reichert WM, Willauer HD, Broker GA, Rogers RD (2001) Characterization and comparison of hydrophilic and hydrophobic room temperature ionic liquids incorporating the imidazolium cation. Green Chem 3:156-164. doi:10.1039/b103275p

28. Shen Y, Zhang Y, Han D, Wang Z, Kuehner D, Niu L (2009) Preparation of colorless ionic liquids "on water" for spectroscopy. Talanta 78:805-808. doi:10.1016/j.talanta.2008.12.056

29. Fujii K, Aruga K, Sekine A, Uekusa H, Sohno K, Tanaka K (2011) In situ observation and mechanistic elucidation of metastable colored species in crystalline state photochromism of trans-biindenylidenedione. Cryst Eng Comm 13:731-733. doi:10.1039/COCE00642D

30. Han J, Wei Y-H, Zhang F-Y, Liu J-Y, Pang M-L, Meng J-B (2009) Synthesis, photochromism and photomagnetism of new biindenylidenedione derivatives in crystalline state. J Mol Struct 920:23-29. doi:10.1016/j. molstruc.2008.10.031

31. Han J, Meng J-B (2009) Progress in synthesis, photochromism and photomagnetism of biindenylidenedione derivatives. J Photochem Photobiol C 10:141-147. doi:10.1016/j.jphotochemrev.2009.10.001 